\title{
Made to measure: Easy Synthesis and Characterization of Nanocomposites with Tailored Functionalities for Chemistry Education
}

\author{
Reza Saadat ${ }^{1, *}$, Björn Bartram ${ }^{2, *}$, Timm Wilke ${ }^{2, *}$ \\ ${ }^{1}$ Institute for Particle Technology, Technische Universität Braunschweig, Germany \\ ${ }^{2}$ Institute of Environmental and Sustainable Chemistry, Department of Chemistry Education, \\ Technische Universität Braunschweig, Germany \\ *Corresponding author: t.wilke@tu-braunschweig.de
}

Received January 10, 2019; Revised February 16, 2019; Accepted March 15, 2019

\begin{abstract}
By adding nanoparticles to classical polymer systems, new functional materials with tailored properties can be obtained. The great variety of possibilities opens up just as many interesting fields of application for industry and science. Following on from this, this article will present how the classical educational subject area of polymers can be extended by a current research context. In a series of experiments, students are using inexpensive chemicals and simple materials from the hardware store to produce nanocomposites that have a much greater hardness or antimicrobial activity than polymers alone. Their properties can then be investigated using a simple test method. Overall, it will be illustrated, that the combination of polymer chemistry and nanotechnology offers a variety of learning opportunities and questions with curricular relevance for schools and student laboratories.
\end{abstract}

Keywords: nanocomposite materials, nanotechnology, polymers, school chemistry education

Cite This Article: Reza Saadat, Björn Bartram, and Timm Wilke, "Made to measure: Easy Synthesis and Characterization of Nanocomposites with Tailored Functionalities for Chemistry Education." World Journal of Chemical Education, vol. 7, no. 2 (2019): 65-71. doi: 10.12691/wjce-7-2-5.

\section{Polymers with Tailor-made Properties}

The use of plastics is being viewed increasingly skeptical by the majority of our society. Since 1950, 8.3 billion tons of plastic have been produced, of which only 600 million tons have been recycled [1]. From a materials science perspective, however, these polymers are functional materials with valuable properties. These properties lead to a wide range of applications that we use and need in our society on a daily basis. The possibility of tailoring the material properties by selecting and cross-linking different monomers has contributed to these technologies. Transparent or opaque, flexible or rigid, biodegradable or stable - desired duro- or thermoplastics, elastomers - or even mixed forms - can be produced for almost any requirement [2].

The choice of monomers or the adjustment of reaction parameters (such as temperature or reaction time) also offer the possibility of tailoring the product properties within certain limits. These parameters influence the degree of crosslinking of the polymerizing monomers, the hardness, physical and chemical stability of the product. In many cases, however, modern functional materials are subject to much higher demands, which can only be achieved to some extent by varying these reaction parameters. For this purpose, additives are often applied, i.e. substances, which are added during synthesis in order to enhance the existing properties of the polymers or to supplement new ones. Color pigments (e.g. carbon black or titanium dioxide) and plasticizers, stabilizers against the effects of UV light (benzophenones) and heat $(\mathrm{Ca} / \mathrm{Zn})$ are well-known examples from industry.

The homogenous distribution of these additives embedded in the polymer represents in many cases a technical challenge for production. In general, processing is easier the smaller the particles are. This is one of the reasons why additives are often applied as nanoparticles. In comparison to macroscopic large ("bulk") particles ...

- the drastically increased surface-to-volume ratio of nanomaterials can significantly reduce the amount of additive required,

- the small particle diameter allows a more homogeneous distribution of the additives in the polymer and

- furthermore a better embedding within the polymer.

Finally, yet importantly, nanomaterials often provide the compound with highly interesting properties that cannot be achieved with bulk materials. This will be illustrated in the following by the example of breakresistant and antimicrobial composite materials. 


\section{Scientific Background and Didactical Reconstruction}

\section{Break-Resistant Composite Materials}

An important field of research at the interface of chemistry and material sciences is composite lightweight construction. Lighter materials with high mechanical strength are of great interest for many branches of industry (e.g. automotive or aerospace industry), as they can save costs and reduce fuel consumption.

The Institute for Particle Technology (iPAT) at the Technische Universität Braunschweig is currently focusing on the development of such materials. By incorporating functionalized boehmite nanoparticles $(\gamma-\mathrm{AlOOH})$ as an additive in a polymer system, the resulting composite shows a much greater hardness than pure polymer. The reason for this is the fine distribution of the boehmite in the material and the hardness of this additive itself (Figure 1, left).

Another important influencing factor is the interaction between polymer and additive. If the latter are not only finely distributed in the matrix but also covalently bonded to the polymer via functionalized surfaces, the crosslinking of the particles with the surrounding matrix - and thus also the formation of cracks and scratches - can be controlled even better (Figure 1, right). Expressed in numbers, the scratch depth in the composite can be reduced by up to $50 \%[3,4,5]$. As indicated in section 1, the advantage of using nanoparticles is that boehmite can be integrated into the polymer matrix more finely distributed. This significantly increases the probability that crack propagation in the material can be better controlled [4,5].

\section{Antimicrobial Composite Materials}

Silver-based antimicrobial agents are of great interest in application areas with special hygienic requirements and demands. These include hospitals, medical practices and the food processing industry on the one hand and door knobs, stair railings and many more on the other. Furthermore, silver nanoparticles are used in other products - silver-containing washing machines, refrigerators, toothpaste, cleaning rags, clothing and plasters are just a few examples. The active element are actually the silver ions that are released from the silver surface over time. Due to their large surface-to-volume ratio, silver nanoparticles tend to increased oxidation and thus the formation of silver ions, making them effective antimicrobial agents. For this reason, they are preferably applied. Because of its frequent use, the annual production of silver nanoparticles in 2012 already amounted to 55 tons and is continuously increasing [6].

Many materials consist of plastic - in combination with silver nanoparticles, new fields of application can be opened up with little material usage. By skillfully embedding the silver nano-additives, a depot effect with long, continuous material release can be achieved, which offers advantages over a simple coating in terms of stability $[7,8]$. An overview with many more information and examples can be found in [9].

However, the use of silver nanoparticles is controversially discussed. Increased use results in increased input into the environment. In 2015, 3.9 tons of silver nanoparticles from toothpaste were discharged into wastewater in the United States alone [10], leading to direct consequences for the ecological balance in water bodies and unknown long-term consequences for animals and humans with regard to accumulation of silver in the food chain.

\section{Didactical Reconstruction}

The synthesis of polymers with nanoadditives offers great didactic potential in several respects and many learning opportunities for teaching in schools and student laboratories. Plastics and polymers are firmly anchored in all K-12 school curricula throughout Germany and are subject of many didactic publications [11-15]. Following on from this, we would like to present experimental approaches for synthesis and characterization of a comparable composite and describe how these classical contents of chemistry teaching can be enriched by the consideration of nanoadditives in current research contexts, questions and methods.

At the research methodological level, the Model of Educational Transfer serves as a theoretical framework for the implementation of this process [16]. Based on the Model of Educational Reconstruction [17], didacticians and chemists cooperate in order to collect subject-specific concepts and student perspectives and to place them in a meaningful relationship to one another. On this basis, the teaching units are didactically designed and structured. The developed materials and experiments are then tested in school laboratories and at partner schools. Teachers and students are involved in order to strengthen the link with teaching practice and later act as multipliers. In a cyclic process of conception, testing, evaluation and optimization, the materials are improved and then disseminated into school practice (see Figure 2).

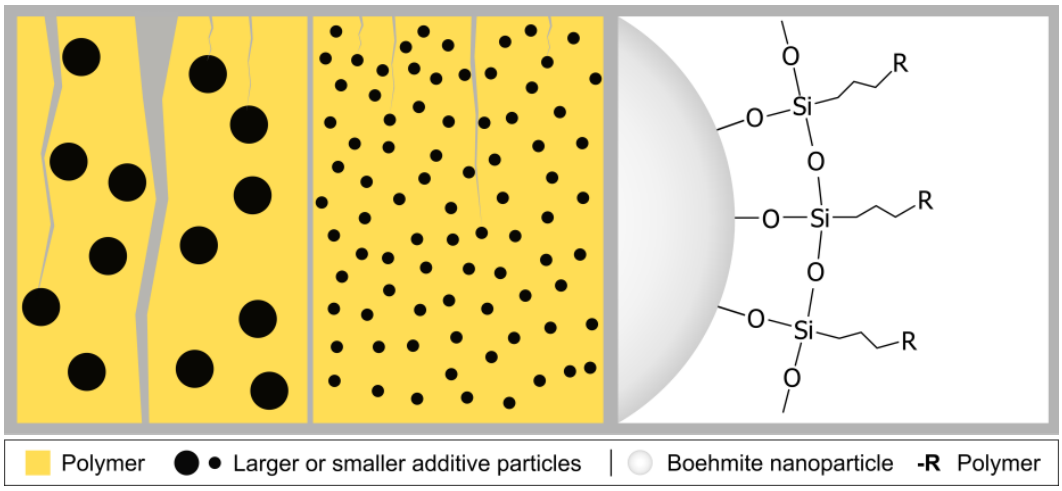

Figure 1. Model representation of the distribution of additives with larger and smaller particle diameters within a polymer with comparative representation of crack formation and propagation (left). Covalent between nanoparticle and polymer through functionalized surfaces (right) 


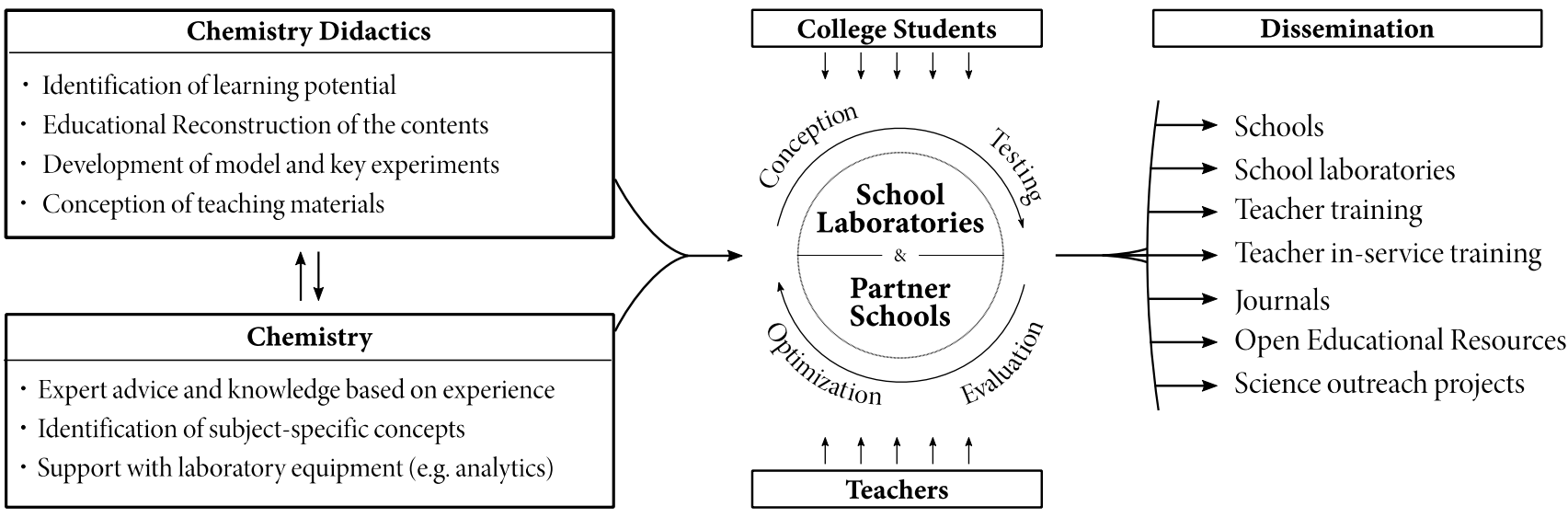

Figure 2. Educational Transfer Research Model [16]

This paper describes selected steps of the process. The following chapters three and four present simple experimental approaches for the synthesis and characterization of fracture-resistant and antimicrobial nanocomposites that can be performed with simple and inexpensive materials and chemicals. In the concluding fifth chapter, we will briefly illustrate how this interesting topic can be implemented in schools and school laboratories.

\section{Synthesis and Characterization of Break-resistant $\mathrm{Al}_{2} \mathrm{O}_{3}$-Nanocomposites}

\subsection{Synthesis}

Research at iPAT uses a specialized polymer system for the synthesis of functional fiber reinforced composites for lightweight construction. The disadvantage, however, for chemistry teaching is the difficult accessibility of the starting materials and a sophisticated temperature control that is required during the curing process. For this reason, a simple resin from the hardware store was chosen instead for the following experiments; the same reason also led to the use of aluminium oxide nanoparticles instead of boehmite. Aluminium tea light dishes are employed as reaction vessels to obtain comparable test specimens for each synthesis in the test series without having to destroy glassware. Experiment 1 describes the synthesis of nanocomposites; a material list with sources of supply can be found in the online supplement to this article.

\section{Experiment 1: Synthesis of Aluminium Oxide}

\section{Nanocomposites}

Equipment and chemicals: 2-component polyester resin ("Presto polyester resin”), aluminium oxide nanoparticles, beaker, tea light shell, spatula, magnetic stirrer with stirring magnet or glass rod, tweezers.

Procedure: $20 \mathrm{~g}$ resin (component 1) and then 4 mass\% of the hardener (component 2, here: $0,8 \mathrm{~g}$ ) are placed in a beaker while stirring to form the basis for the production of four specimen. After three minutes, the stirring magnet is removed and the samples are immediately prepared as follows.

For Sample 1 (reference), $10 \mathrm{~g}$ of the mixture are placed in a tea light shell.

For Sample 2, 0,5 g aluminium oxide nanoparticles are gradually added to the remaining $10 \mathrm{~g}$ of the mixture in the beaker. Immediately afterwards, and the dispersion is stirred for two more minutes and placed in a tea light shell. If a stirring magnet was used, it is now removed.

Both samples are labelled and left to harden overnight.

Observation: Both dispersions are yellowish clear. After the hardener has been added, the dispersion gradually turns brownish, and within 30 minutes, the mixture heats up and begins to harden. The samples without nanoadditives are clear after hardening, the samples with nanoadditives are opaque (see Figure 3).
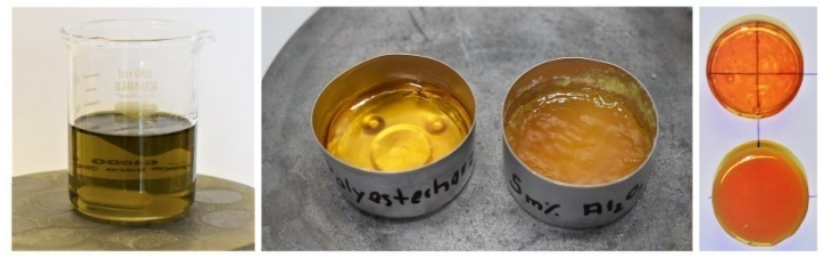

Figure 3. Preparation of the basic mixture (left); hardening of polymer and nanocomposite in tea light shells (center); transparent polymer and opaque nanocomposite (right)

Analysis: The resin essentially consists of styrene (more precisely: various isomers of methyl styrene and styrene), the hardener (dibenzoyl peroxide) is classically used as a radical starter due to its low stability. Due to the decomposition of dibenzoyl peroxide, phenyl radicals are formed which serve as starters of the chain reaction. In the subsequent radical polymerization, a chain growth takes place by the addition of further monomers: polystyrene is formed (see Figure 4).

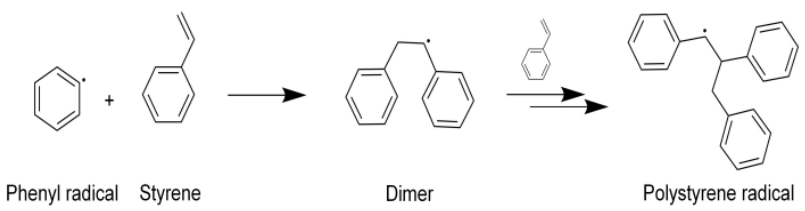

Figure 4. Mechanism for the synthesis of polystyrene via radical polymerization

$\mathrm{Al}_{2} \mathrm{O}_{3}$ nanoparticles are dispersed at the beginning of the reaction and are embedded into the polymer matrix during the course of the reaction. The exact nature of the interaction between $\mathrm{Al}_{2} \mathrm{O}_{3}$ nanoparticles has not yet been conclusively clarified and is the subject of current research [5]. 


\subsection{Characterization of Breaking Strength}

Optical differences between the two samples are hardly noticeable at first glance. In the following, both specimens will be tested for hardness in Experiment 2 using a simple test procedure.

Material sciences and testing institutes have various standardized methods for testing the toughness of materials. These include the Charpy impact test for testing breaking strength (cf. DIN EN ISO 179-1) or tensile testing, in which a sample is subjected to a controlled tension until failure (cf. ISO 527). Both represent standardized test methods, which, however, require precise and complex apparatus or sample forms.

A low-cost-test method for school chemistry education should ideally be as precise, simple, harmless, inexpensive, reproducible and - if such an evaluation is desired - quantifiable. Such a method can certainly be developed in various ways by the teacher or in cooperation with the learning group. An in-depth didactic examination of this topic with examples and teaching materials can be found in Sommer et al. [18].

One example of testing the hardness of the specimen is the (repeated) effect of an identical mechanical load on the polymer. For this purpose, a metallic ball with a known mass is dropped from a known height onto the sample. In order to increase the reproducibility of the method, a simple plastic pipe from a hardware store can be used as a downpipe. In this way, it is possible to compare the height at which the polymer sample breaks due to the impact.

\section{Experiment 2: Simple Breaking strength test}

Equipment and chemicals: samples 1 and 2 from experiment 1, downpipe (approx. $1 \mathrm{~m}$ long, $50 \mathrm{~mm}$ in diameter), iron ball, drill bit, nail, wooden plate (optional).

Procedure: In preparation, holes (here: $\varnothing=4 \mathrm{~mm}$ ) are drilled in the downpipe at intervals of $5 \mathrm{~cm}$. A nail is inserted through the opening at the desired height to hold the ball in position.

The reference (sample 1) is placed on a wooden board to protect the ground. The ball is placed in the downpipe and held at the desired height with the nail. The downpipe is placed over the sample and the ball is dropped onto the sample by pulling the nail (see Figure 5). This step is repeated while raising the drop height gradually until the sample breaks. Afterwards, the test series is repeated with sample 2.

Observation: Material samples without $\mathrm{Al}_{2} \mathrm{O}_{3}$ nanoparticles break at significantly lower drop heights or show more chipping and cracking than samples with added nanoadditives. The ball which was used $(\mathrm{m}=372.2 \mathrm{~g}, \varnothing=4.5 \mathrm{~cm})$ broke sample 1 at a drop height of $0.6 \mathrm{~m}$, sample 2 at $1.10 \mathrm{~m}$.

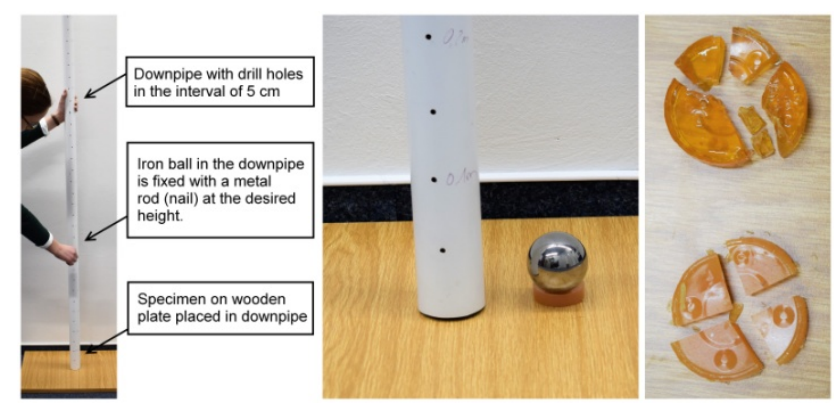

Figure 5. Simple characterization method (left); broken polymer and nanocomposite samples (right).
Analysis: The polymer samples are mechanically loaded by the impulse of the ball, which is shown by crack formation, splintering or fragmentation. In comparison to the untreated polymer, the $\mathrm{Al}_{2} \mathrm{O}_{3}$ nanoadditives reduce this crack formation, so that the functionalized material resists greater loads (see section 2).

This test procedure reflects the interdisciplinary character of the material sciences: On the one hand, the interval halving method from mathematics offers the possibility of efficiently determining the correct drop height with as few samples as possible. From a physical perspective, the final velocity $(\mathrm{v})$ of the metallic ball and thus the momentum (p) can be easily calculated by weighing the ball $(\mathrm{m})$ together with the measured drop height (h) and the acceleration due to gravity (g) using the laws of the free fall.

$$
\begin{gathered}
\mathrm{v}=\sqrt{2 \cdot \mathrm{g} \cdot \mathrm{h}} \\
\mathrm{p}=\mathrm{m} \cdot \mathrm{v}
\end{gathered}
$$

Thus the difference can be quantitatively described in Ns; for sample 1 it is 1.28 Ns, for sample 21.73 Ns. This corresponds to an increase of about $35 \%$.

Overall, the results clearly show that a significant increase in material hardness can be achieved by adding a small amount of $\mathrm{Al}_{2} \mathrm{O}_{3}$ nanoadditive to the polymer.

Yet, one question remains unanswered: Does it have to be nanoparticles? Interested learning groups can take up this expected student question and investigate the influence of particle size. By comparing aluminium oxide with macroscopic, microscopic and nanoscale particle sizes, simple structure-property relationships can be derived on the basis of the comparative examination of the test results.

\section{Synthesis and Characterization of Antimicrobial Silver-Nanocomposites}

As mentioned above, silver-containing materials are used for their antimicrobial effect. It is often desired (e.g. in refrigerators, food storage containers, ...) that the products maintain their effect for a long time. Similar to drugs, silver nanoparticles or ions should ideally be emitted in small quantities over a longer period of time. In this section, this requirement and thus a typical material science challenge will be addressed. Specifically, the production and lifetime of nanosilver-coated materials and nanosilver composites will be compared within a model experiment.

\subsection{Synthesis of Nanosilver-coated Polymers and Silver Nanocomposites}

Silver can be purchased as nanopowder from numerous suppliers, but the average price of $60 €$ for 5 grams is fairly high. It is much less expensive to synthesize the required particles by yourself. For this, the synthesis according to Wang [19] with changes according to Kittler [20] can be carried out. In this way, a sufficient amount of a silver nanoparticle dispersion is easily obtained, which will then be applied to coat polymer plates and to fabricate nanocomposites. 
Experiment 3: Silver nanoparticle synthesis and preparation of polymers/composites

Equipment and chemicals: Glucose (Roth), Polyvinylpyrrolidon (Sigma Aldrich; short: PVP), silvernitrate solution ( $0.1 \mathrm{M}$, Grüssing), 2 polymer plates (from experiment 1), beakers $(250 \mathrm{~mL})$, heating plate with magnetic stirrer, thermometer, spatula, pipette/measuring cylinders, drying furnace, tweezers

Procedure: For the synthesis of a silver nanoparticle dispersion, 2 g glucose and $1 \mathrm{~g}$ PVP are placed in a beaker and dissolved in $160 \mathrm{~mL}$ water. The solution is heated to $90^{\circ} \mathrm{C}$. When the temperature is reached, 29.4 $\mathrm{mL}$ of the silver nitrate solution were are added while stirring vigorously. The dispersion is stirred at $90{ }^{\circ} \mathrm{C}$ for 1 hour before it was is slowly cooled to room temperature without further stirring. During the synthesis, the initially colorless, clear solution turns yellowish over time after the addition of the silver nitrate, indicating the formation of silver nanoparticles. A longer reaction can result in brown discoloration or even greyish/silver residue.

For preparation of a polymer coated with silver nanoparticles, one polymer plate from experiment 1 is tied with a string and hung in a $100 \mathrm{~mL}$ beaker with the aid of a wooden rod (see. Figure 6, left). There it is poured with the silver nanoparticle dispersion until it is just covered. Then the beaker with the polymer plate is placed in the drying oven at $90^{\circ} \mathrm{C}$ overnight. After drying, the coated polymer plate is removed and stored for experiment 4.1.

For the preparation of composites with embedded silver-nanoparticles (silver nanocomposites), the sediment is removed from the very same beaker with a spatula and, if necessary, mortared. The solid obtained is then used as a nanosilver additive for the synthesis of silver nanocomposites, which is carried out according to the same procedure as in experiment 1 . The quantities for this shall be chosen in a way that the percentage by mass of the silver is (at least) 1 mass $\%$.

Observation: After the solvent of the silver nanoparticle dispersion has evaporated overnight, an orange-black solid remains as a residue. This is clearly visible as a coating on the polymer plate (Figure 6, center).

The silver nanocomposites produced with the residue do not differ significantly from the pure polymer plates, but have small visible inclusions inside (Figure 6, right).

Analysis: During the course of the reaction, silver ions are reduced to elemental silver by glucose.

$$
\begin{aligned}
2 \mathrm{Ag}^{+}+ & \mathrm{C}_{5} \mathrm{H}_{11} \mathrm{O}_{5}-\mathrm{COH}+2 \mathrm{OH}^{-} \rightarrow \\
& 2 \mathrm{Ag}+\mathrm{C}_{5} \mathrm{H}_{11} \mathrm{O}_{5}-\mathrm{COOH}+2 \mathrm{H}_{2} \mathrm{O}
\end{aligned}
$$

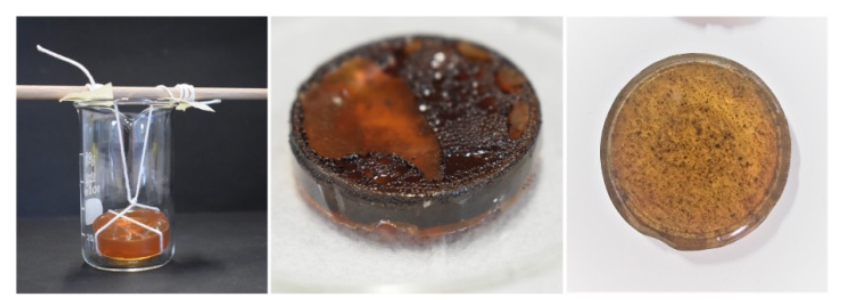

Figure 6. Fabrication of polymer coated with silver nanoparticles (left, center); silver nanocomposite with visible inclusions (right)

The resulting silver is surrounded by PVP and thus stabilized in such a way that the generated nanoparticles are inhibited in their agglomeration or growth. The color serves as an indicator of the size: yellow tones indicate nanoparticles; grey tones indicate larger particles. More detailed information on the underlying mechanism and stabilization can be found at [19].

As the solvent evaporates, PVP coated silver nanoparticles remain on the beaker and the polymer plate. Despite the stabilizing effect of PVP, the temperature and volume constriction can cause agglomerates, which can be recognized by the corresponding color deviations.

Compared to the synthesis of aluminium oxide nanocomposites, where no white agglomerates are visible, the dried silver nanoparticles can be recognized as black agglomerates in the final composite. The reason for this may be the lack of solubility of the PVP in the nonpolar resin, which counteracts dispersion. The glucose residues and gluconic acid contained in the dispersion probably also counteract dispersion due to their poor solubility. This condition impedes a completely homogeneous distribution of the additive within the polymer, but does not pose a problem for the subsequent experiment.

\subsection{Emission and Comparison of Antimicrobial Activity}

Due to the good redispersibility of the synthesized silver nanoparticles, it is easy to compare the emission of coated polymers and nanosilver composites. This can be achieved in many ways using classical analytical methods, e.g. photometry or Volhard titration. Thanks to the intensive coloration of the nanosilver dispersion, semiquantitative analysis is already possible with the naked eye, which makes it easier to carry out in chemistry class (Experiment 4.1).

\section{Experiment 4.1: Coating vs. Composite - Comparison} of nanosilver emissions

Equipment and chemicals: Nanosilver coated polymer plate, nanosilver composite (both from experiment 3), beaker $(100 \mathrm{~mL})$, tweezers, measuring cylinder

The beakers are each filled with $20 \mathrm{~mL}$ of water. A specimen (coated plate or nanosilver composite) is placed in a beaker for one minute and swivelled slightly. Then it is removed with tweezers and rinsed with water. The specimen is then placed in the next beaker with $20 \mathrm{~mL}$ water - this process is repeated until no more optical changes can be observed in the solution.

Observation: For the coated polymer, a dark red-brown coloration of the water is observed after the first wash; during the second wash, however, no coloration is visible (see Figure 7, left). Further, the coating on the surface of the polymer disappears.

In the case of nanocomposite, the water turns yellow for several washes; after an average of eight repetitions, the obtained solution is almost colorless (see Figure 7, right).

Analysis: With the coated polymer, the nanoparticles adhere only weakly to the surface and can thus also be easily removed mechanically. In contact with water, the soluble residues easily dissolve again and the nanoparticles are dispersed. This results in a strong colouring of the water on one side and a lack of colouring in further washing cycles on the other side. 

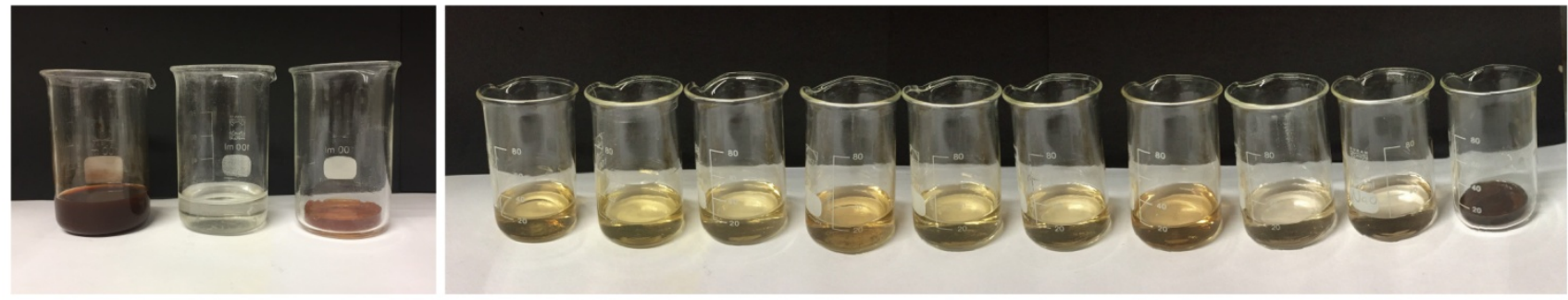

Figure 7. Silver nanoparticle emissions from polymers coated with nanoparticles (left) and silver nanocomposites (right)

In the case of nanocomposites, the silver nanoparticles (or smaller conglomerates) are incorporated directly into the polymer matrix. Since the particles are partially enclosed and thus only indirectly accessible, the emission is delayed. Due to this depot effect, the nanoparticles are not released immediately but continuously over a longer period of time.

Within the last Experiment 4.2, the antimicrobial activity of the obtained solutions shall be compared. The effect on the activity of yeast as described by Dege [21] is a simple method with a direct relation to student's day-to-day lives well suited for chemistry teaching. The results with the solutions obtained in 4.1 are described in the following.

\section{Experiment 4.2: Antimicrobial effect of silver} nanoparticles on yeast activity

In the following, the antimicrobial effect of the nanosilver dispersions will be investigated quantitatively with the aid of a gas syringe.

Equipment and chemicals: Nanosilver dispersions (from Experiment 4.1), glucose (Roth), dry yeast, Gas syringe $(100 \mathrm{~mL})$, beaker, crystallizing bowl, thermometer, Erlenmeyer flask (wide neck), plug with two drill holes, rubber hose, glass tube, heating plate with magnetic stirrer, stirring plates, stirring magnet, hose clamps

Procedure: The experiment is set up as shown in Figure 8. The water bath is then heated to $35^{\circ} \mathrm{C}$ and a 1 mass\% sugar solution is prepared during the waiting time. Once the water bath is heated, $1.4 \mathrm{~g}$ dry yeast is placed in an Erlenmeyer flask with a wide opening and dispersed in $20 \mathrm{~mL}$ of the sugar solution. The silver-containing solution to be tested is then quickly added and sealed with the plug. In order not to distort the measurement, it should be ensured that the valve is opened when the plug is closed and closed afterwards.

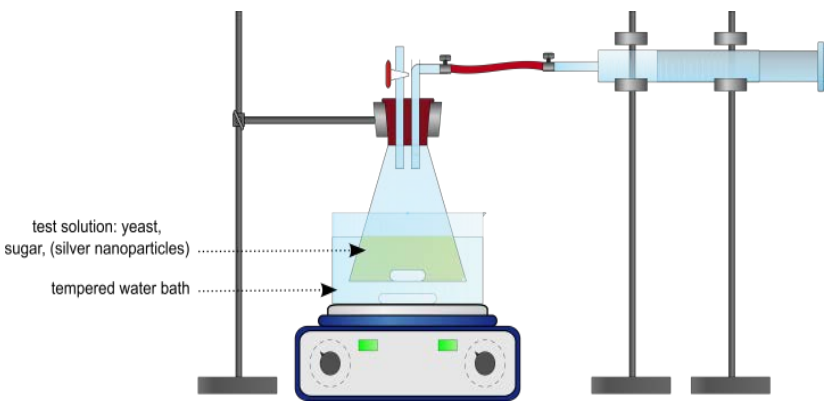

Figure 8. Schematic representation of the setup for quantitative measurement of yeast activity

The gas development on the gas syringe is then measured and recorded every 30 seconds over a period of 20 minutes. This procedure is repeated for each silver-containing solution and once without the addition of silver as a reference.

Observation: A gas development can be observed after only a few minutes, but it varies depending on the solution investigated. The corresponding results are shown in Figure 9.

Analysis: The resulting carbon dioxide serves as an indicator of yeast activity. The results show a decreasing yeast activity with all silver-containing solutions due to the biocidal effect of silver, more precisely silver ions. A visible correlation between the intensity of the coloring of the nanosilver dispersion and the measured yeast activity can be observed. For a more detailed investigation of the effectiveness of silver ions and nanoparticles, see [22].

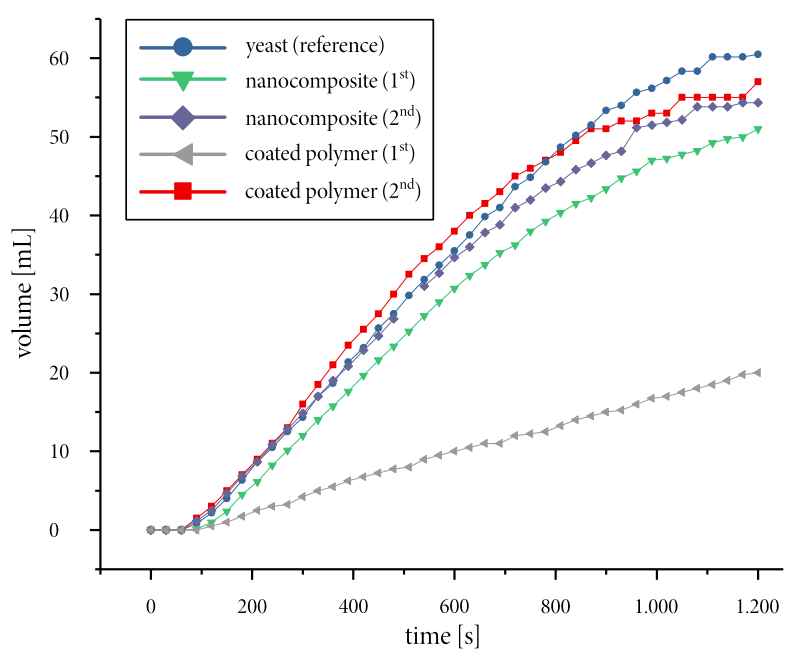

Figure 9. Yeast activity with different sample solutions according to the produced $\mathrm{CO}_{2}$ volume

The test series can only be understood as a model experiment, which can still be extended in many respects. For example, the effect of PVP is neglected for didactic reasons. Furthermore, by varying the parameters (e.g. reducing the amount of yeast, increasing the amount of silver) the differences of the results can be worked out even more clearly.

\section{Implementation into School Chemistry Education}

Polymers and plastics are currently an integral part of chemistry education in many countries. This topic, which is highly relevant to everyday life, can be used to interconnect a wide range of important chemical contents, such as reaction mechanisms, synthesis concepts and 
elementary structure-property relationships. The recycling of plastics can further be used as an example of material cycles in everyday life; numerous media reports on plastics in the world's oceans also offer opportunities to promote assessment competencies and, last but not least, to reflect on one's own behavior. However, the ability to specifically design the material properties of polymers is often neglected. While widely used plastics (polyethylene, polypropylene, polyvinyl chloride, polystyrene, ...) are presented, reaction parameters, additives and functionalization tend to receive less attention. Nevertheless, these are indispensable for the production of modern functional materials with precise requirements. In addition, the production of composites is easy to understand and to implement experimentally.

This contribution is intended as an encouragement to discuss the fabrication, characterization and application of nanocomposites in school chemistry education. The experiments described in this contribution provide a starting point for this, that can be extended in many ways - for example by using other additives such as (fluorescent) zinc oxide nanoparticles, (UV-absorbing) titanium dioxide nanoparticles, (heat resistant) copper nanoparticles and many others. In seminar papers or student projects, other parameters can also be varied, such as the polymer used or the amount of additive used. In this way, students can use variable control strategies to independently develop a series of experiments to optimize their composite and then evaluate the products from different perspectives (materials science, economics).

Overall, these aspects offer a wide range of opportunities to expand the classic subject area of plastics and polymers to include current research findings and contexts. The large number of innovations in materials science in recent years is excellently suited to building a bridge between research and the world in which students live by means of major projects from the aerospace industry and everyday products.

\section{Acknowledgements}

We acknowledge support by the German Chemical Industry Fund (FCI), the German Research Foundation and the Open Access Publication Funds of the Technische Universität Braunschweig.

\section{References}

[1] Geyer, R., Jambeck, J. R., Law, K. L. (2017). Production, use, and fate of all plastics ever made. Science advances 3/7, e1700782.

[2] Huntemann, H., Vennemann, Hendrik, Parchmann, Ilka (2000). Ein Auto ohne Kunststoffe? Eine Unterrichtseinheit aus der Konzeption Chemie im Kontext. Praxis der Naturwissenschaften - Chemie 49/4, $19 \mathrm{ff}$
[3] Sangermano, M., Messori, M. (2010). Scratch Resistance Enhancement of Polymer Coatings. Macromol. Mater. Eng. 295/7, 603-612.

[4] Saadat, R., Kockmann, A., Garnweitner, G. (2017). Tailored Surface Modification of Nanoparticles for Nanocomposites with Optimized Mechanical Properties. Paris.

[5] Kockmann, A., Porsiel, J. C., Saadat, R., Garnweitner, G. (2018). Impact of nanoparticle surface modification on the mechanical properties of polystyrene-based nanocomposites. RSC Advances 8/20, 11109-11118.

[6] Piccinno, F., Gottschalk, F., Seeger, S., Nowack, B. (2012). Industrial production quantities and uses of ten engineered nanomaterials in Europe and the world. J Nanopart Res 14/9, 300.

[7] Block, S. S. (1983). Disinfection, sterilization, and preservation, 3. Ed. Lea \& Febiger, Philadelphia Pa.

[8] Slawson, R. M., Lee, H., Trevors, J. T. (1990). Bacterial interactions with silver. Biol Metals 3/3-4, 151-154.

[9] Elias, H.-G. (2000). Plastics, General Survey.

[10] Junevičius, J., Žilinskas, J., Česaitis, K., Česaitienė, G., Gleiznys, D., Maželienè, Ž. (2015). Antimicrobial activity of silver and gold in toothpastes: A comparative analysis. Stomatologija 17/1, 9-12.

[11] Kröger, S., Hock, K., Tausch, M., Anton, M., Bader, A.,

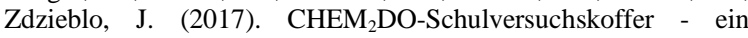
Kooperationsprojekt von Wirtschaft, Fachdidaktik und Lehrerfortbildungszentren. CHEMKON 24/4, 241-245.

[12] Banerji, A., Tausch, M. W., Scherf, U. (2012). Fantastic Plastic. CHEMKON 19/1, 7-12.

[13] Stäudel, L., Schmidkunz, H., Rau, T. (2010). Von linear bis hochvernetzt - Struktur-Eigenschafts-Beziehungen am Beispiel Kunststoffe. Unterricht Chemie 21/115, 38-42.

[14] Bader, H.-J., Sgoff, D. (2007). Schulversuche zu faserverstärkten Kunststoffen. Praxis der Naturwissenschaften - Chemie 56/7, 19-23.

[15] Huntemann, H., Parchmann, I. (2000). Biologisch abbaubare Kunststoffe Einordnung in ein neues Konzept für den Chemieunterricht. CHEMKON 7/1, 15-21.

[16] Bartram, B., Wilke, T. (2018). Aus der Forschung in die Schule Gestaltung einer lernortübergreifenden Summer School zum Thema Nanotechnologie durch Fachdidaktische Transferforschung. In: Theorie und Praxis im Spannungsverhältnis. Beiträge für die Unterrichtsentwicklung, 1. Ed. Fereidooni, K., Hein, K., Kraus, K. (Eds.). Waxmann, Münster, 67-82.

[17] Duit, R., Gropengießer, H., Kattmann, U., Komorek, M., Parchmann, I. (2012). The Model of Educational Reconstruction a Framework for Improving Teaching and Learning Science1. In: Science Education Research and Practice in Europe. Retrospective and Prospective. Jorde, D., Dillon, J. (Hrsg.). Sense Publishers, Rotterdam, 13-37.

[18] Sommer, K., Krupp, U., Toschka, C., Schröder, T. P. (2017). Für Lernprozesse modelliert. Physikalisch-chemische Prüfverfahren der Industrie an den Beispielen Klebefestigkeit, Viskosität und Waschleistung. CHEMKON 24/4, 227-232.

[19] Wang, H., Qiao, X., Chen, J., Ding, S. (2005). Preparation of silver nanoparticles by chemical reduction method. Colloids and Surfaces A: Physicochemical and Engineering Aspects 256/2-3, 111-115.

[20] Kittler, S., Greulich, C., Gebauer, J. S., Diendorf, J., Treuel, L., Ruiz, L., Gonzalez-Calbet, J. M., Vallet-Regi, M., Zellner, R., Köller, M., Epple, M. (2010). The influence of proteins on the dispersability and cell-biological activity of silver nanoparticles. J. Mater. Chem. 20/3, 512-518.

[21] Dege, J., Haffer, S., Waitz, T. (2016). Sind Nanopartikel schädlich für Mikroorganismen? Experimente zur Toxizität von Nanopartikeln im Chemieunterricht. Unterricht Chemie 2, 28-33.

[22] Kędziora, A., Speruda, M., Krzyżewska, E., Rybka, J., Łukowiak, A., Bugla-Płoskońska, G. (2018). Similarities and Differences between Silver Ions and Silver in Nanoforms as Antibacterial Agents. International journal of molecular sciences 19/2. 